remaining $21.5 \%$ reported improvement in severity of disease. Thus, we strongly advocate that nutrition therapy should at least be offered as a treatment modality to every Crohn's patient during gastroenterology consultations.

\section{PTH-29 INTENTIONAL FOREIGN BODY INGESTION DURING THE COVID-19 CRISIS: A CRY FOR HELP?}

Luke Hanna*, Nina-Joyce Shehata, Tracie Jarvis, Fergus Thursby-Pelham, Sharmila Subramaniam. Department of Gastroenterology, Portsmouth Hospitals University NHS Trust, UK

\subsection{6/gutjpl-2021-BSG.344}

Introduction Endoscopy was significantly affected by the COVID-19 pandemic in the past year, meaning any increased demand placed significant strains on services. Rising rates of intentional foreign body ingestion (IFBI) requiring urgent endoscopy impacted on our centre in this period leading us to compare rates with pre-pandemic levels and analyse it against key dates in the UK coronavirus timeline.

Methods Endoscopic records in a large acute NHS trust in South England were examined retrospectively between 01/01/ 2019 - 31/12/2020 to identify gastroscopies for foreign body removal. Procedures were reviewed and individuals' records interrogated to identify information on their backgrounds and prior behaviours. Rates of IFBI were examined against contemporary events of the COVID pandemic.

Results 122 endoscopies were identified for foreign body removal. Of these: $46(37.7 \%)$ were for IFBI, 69 (56.6\%) for food bolus removal, and 7 (5.7\%) for accidental foreign body ingestion. Whilst rates for food bolus dropped by almost a third in 2020 (compared to 2019), we noted an 8.2 times increase in IFBI procedures for the same period. 8 individual patients contributed to the IFBI workload. $54.3 \%$ of IFBI endoscopies required general anaesthetic and 95\% involved sharp or dangerous objects.

Rates were analysed against the COVID timeline (Table 1). Between 01/01/2020 - 23/03/2020, when global COVID alerts were rising, levels of IFBI were relatively high with 5 procedures performed (equal to total 2019 number). From 23/03/ 2020-10/05/2020, when the UK was in its 1st lockdown, numbers dropped with only one case recorded. After this point levels rose dramatically with 1.13 procedures a week logged until 31/10/2020, when the UK re-entered lockdown. The 2nd lockdown, and later tiered system, did not see dramatic reductions in activity as cases continued at 0.80 week until the end of 2020 .

Conclusions There has been a large increase in IFBI requiring emergency endoscopy in 2020. Mental health stresses during the pandemic seem to have had a role in susceptible individuals reaching crises point in this behaviour. Sustained effects of increased anxiety, in combination with patchy access to mental health services, may have led to this manifestation of selfharm. This represents yet another unforeseen consequence of the COVID pandemic that has affected the utilisation of endoscopy services locally.

\section{PTH-30 THE FIRST UK DIETITIANS PROVIDING ADVANCED PRACTITIONER GI SERVICES}

Anupam Rej* ${ }^{*}$ Rachel Buckle, Anupam Rej, Kumar Basu, Neil Thompson, Nicola Moran, Nick Trott, Imran Aziz, David Sanders. Academic Unit of Gastroenterology, Sheffield Teaching Hospital NHS Foundation Trust, Sheffield, UK

\subsection{6/gutjnl-2021-BSG.345}

Introduction Clinical demand for gastrointestinal (GI) services in the United Kingdom (UK) has grown exponentially and the mean annual consultant expansion of $5 \%$ over the last 10 years has still not dealt with the unmet need. ${ }^{1}$ As a result $43 \%$ of new consultant appointments are vacant and private contractors are filling this space. ${ }^{1}$ We provide data on the first UK GI dietitians to deliver an advanced practitioner role using a payment by results model $(\mathrm{PbR})$.

Methods Two Band 6 dietitians were trained by a physician over a 12-month period (cost $£ 40,500$ per dietitian per annum) to undertake outpatient medical gastroenterology clinics. In addition, they were enrolled into a MSc in advanced clinical practice (cost $£ 8490$ per dietitian). Outpatient clinics assessing patients with symptoms compatible with irritable bowel syndrome (IBS) were performed, initially physician-led, followed by dietetic-led. Patients were invited to assess their clinic satisfaction on a scale from 0-10. Clinical notes were reviewed subsequently to see the diagnostic outcomes of these patients. Cost analysis of income generation from clinics was also performed.

Results 91 clinical notes were reviewed (mean age 38 years, $66 \%$ female $[n=60])$, with $73 \%(n=66)$ having a diagnosis of IBS, $12 \%[\mathrm{n}=11]$ bile acid diarrhoea, 4\% [n=4] functional diarrhoea, 2\% [n=2] microscopic colitis and 9\% $[n=8]$ having another diagnosis. 62\% $[\mathrm{n}=56]$ had been seen by a physician, with $38 \%[n=35]$ being seen by a dietitian. There was no statistically significant difference in clinic satisfaction between physician and dietetic-led clinics (physician-led mean 9.2 \pm 1.5 vs dietitian-led mean $9.4 \pm 1.1, p=0.50)$. Annual income generation was calculated on the basis of 84 clinics annually per dietitian, with 3 new patients and 7 follow ups (total income generation of $£ 68.2 \mathrm{k}$ [ $£ 35.3 \mathrm{k}$ new patients, $£ 32.9 \mathrm{k}$ follow ups]). The cost to employ a band 7 dietitian to deliver this service is $£ 53.5 \mathrm{k}$ (midpoint of scale), providing an estimated net surplus of $£ 14.7 \mathrm{k}$ based on the $2020 / 21 \mathrm{PbR}$ tariff to the directorate.

Conclusions Dietetic-led outpatient clinics for IBS patients can lead to an annual surplus of $£ 14.7 \mathrm{k}$ per dietitian, with no difference in patient satisfaction between physician and dietitian-

Abstract PTH-29 Table 1 IFBI endoscopies against COVID timeline

\begin{tabular}{|c|c|c|c|c|c|c|}
\hline \multirow[t]{2}{*}{ Endoscopies for IFBI } & \multirow[t]{2}{*}{ Total year 2019} & \multicolumn{4}{|l|}{ Jan-Dec 2020} & \multirow[t]{2}{*}{ Total year 2020} \\
\hline & & $\begin{array}{l}01 / 01 / 20-23 / 03 / 20 \\
\text { (pre-lockdown) }\end{array}$ & $\begin{array}{l}23 / 03 / 20-10 / 05 / 20 \\
\text { (1st lockdown) }\end{array}$ & $\begin{array}{l}10 / 05 / 20-31 / 10 / 20 \\
\text { (variable easing of restrictions) }\end{array}$ & $\begin{array}{l}31 / 10 / 20-31 / 12 / 20 \\
\text { (2nd national lockdown }+ \text { tiered restrictions) }\end{array}$ & \\
\hline Total number & 5 & 5 & 1 & 28 & 7 & 41 \\
\hline Procedures per week & 0.10 & 0.43 & 0.15 & 1.13 & 0.80 & 0.79 \\
\hline
\end{tabular}

